\title{
Body weight, serum albumin and food intolerance were linked to upper gastrointestinal Crohn's disease: a 7-year retrospective analysis
}

\author{
Jianfeng Pan^, Dongni Fu, Yong Li, Yifan Wang, Guanghui Lian, Xiaowei Liu \\ Department of Gastroenterology, Xiangya Hospital, Central South University, Changsha, China \\ Contributions: (I) Conception and design: J Pan, Y Li, X Liu; (II) Administrative support: J Pan, G Lian, X Liu; (III) Provision of study materials or \\ patients: J Pan, Y Li, D Fu; (IV) Collection and assembly of data: J Pan, Y Li, Y Wang, G Lian; (V) Data analysis and interpretation: Y Wang, Y Li, \\ D Fu; (VI) Manuscript writing: All authors; (VII) Final approval of manuscript: All authors. \\ Correspondence to: Xiaowei Liu, MD, PhD; Guanghui Lian, MD. Department of Gastroenterology, Xiangya Hospital of Central South University, \\ Changsha, China. Email: liuxw@csu.edu.cn; Lianhappy@csu.edu.cn.
}

Background: The clinical features of upper gastrointestinal (L4) Crohn's disease (CD) and its subtypes,
along with the associated and nutritional status, remain poorly described. Our aim was to evaluate the clinical
characteristics of L4 CD phenotype and its subtypes at diagnosis, and their relationship with the nutritional
status.

Methods: A retrospective study was conducted on 869 CD patients diagnosed between 2013 and 2019, and the association between the clinical characteristics and nutritional status of L4 patients was determined using Random forest importance ranking and logistic regression.

Results: The majority of the patients (59.72\%) presented L4 lesions, of which 335, 158 and 26 had proximal ileal, jejunal and esophago-gastroduodenal (EGD) lesions respectively. L4 patients were predominantly male (OR 2.07), smoker (OR 1.80), and had higher body weight and BMI, longer disease course, and stricturing disease (OR 1.88). Furthermore, the serum albumin level, body weight and disease course showed higher MDG in the random forest importance ranking test for L4 CD and L4-proximal ileal types. According to logistic regression, body weight (OR 1.054), disease course (OR 1.010), stricturing behavior (OR 4.998) and tomato intolerance (OR 1.313) were the independent risk factors for L4. In addition, body weight (OR 1.042) and stricturing behavior (OR 3.152) were the relevant factors for proximal ileal subtype, and stricturing behavior (OR 4.206) and perianal disease (OR 0.339) for jejunal subtype.

Conclusions: L4 disease has a higher incidence rate compared to the non-L4 CD, and mainly affects males, and those with prolonged disease course, stricturing behavior, higher weight, BMI, albumin levels and food intolerance (FI).

Keywords: Crohn's disease (CD); features; food intolerance (FI); nutrition; upper gastrointestinal tract

Submitted Mar 05, 2020. Accepted for publication Sep 18, 2020.

doi: 10.21037/atm-20-2212

View this article at: http://dx.doi.org/10.21037/atm-20-2212

^ ORCID: 0000-0002-8044-647X. 


\section{Introduction}

Crohn's disease (CD) is a chronic inflammatory condition of the gastrointestinal tract that mainly affects the terminal ileum. According to the Montreal classification based on the anatomical location, CD is divided into the ileal (L1), colonic (L2), ileocolonic (L3) and upper gastrointestinal (L4) phenotypes $(1,2)$. Recent studies show that the L4 subgroup is heterogeneous in terms of clinical characteristics and prognosis. Although there is no clinical consensus at present, L4 can be further classified into the proximal ileal, jejunal and esophago-gastroduodenal (EGD) subtypes (3-7). The L4-jejunal and L4-proximal ileal disease indicates a higher risk of intestinal surgery, a more aggressive disease and worse prognosis. For instance, jejunal involvement predicts stricturing and multiple abdominal surgeries $(4,6,7)$. However, the nutritional status of the L4 and non-L4 patients, including weight, body mass index (BMI), albumin levels, diet and others, has not been compared. This is relevant since $85 \%$ of the $\mathrm{CD}$ patients awaiting surgery are malnourished due to improper dietary intake and resorption (8-11). Therefore, it is essential to identify the nutritional factors affecting the different phenotypes in order to individualize disease management and therapy.

The aim of this study was to evaluate the clinical characteristics and underlying nutritional factors of L4 $\mathrm{CD}$ phenotype and its subgroups at diagnosis, and identify the correlations. We conducted a retrospective study in accordance with the STROBE reporting checklist (available at http://dx.doi.org/10.21037/atm-20-2212).

\section{Methods}

\section{Patients and study design}

A retrospective study was conducted on patients diagnosed with CD between January 2013 and December 2019 at the Xiangya Hospital Central South University. The inclusion criteria were as follows: (I) initial diagnosis of CD, (II) availability of gastroscopy, ileocolonoscopy, double-balloon enteroscopy or capsule endoscopy and imaging (computer tomography, or magnetic resonance imaging) data, and (III) availability of the complete operation reports in case of diagnosis via surgery. Patients with indeterminate colitis, ulcerative colitis, intestinal tuberculosis, Ischemic enteropathy, infective enterocolitis, Schistosoma, amebic enteropathy or Behcet disease, younger than 14 years or older than 75 years, and positive for Helicobacter pylori infection as per 13C-urea breath test were excluded. Disease location was established by a gastroenterologist, who also classified the patients into L1-L4 types, and further into the L4 subtypes as described above (Figure S1). The study was conducted in accordance with the Declaration of Helsinki (as revised in 2013), and the study approved by the Ethical Committee of the Xiangya Hospital of Central South University (No. 201912457), and informed consent was taken from all the patients.

\section{Data collection}

Demographic and clinical data including gender, weight, $\mathrm{BMI}$, age at onset, duration of disease, smoking history, disease behavior, perianal disease, $\mathrm{CD}$ activity index (CDAI) score, complications and history of surgery were collected. In addition, laboratory test results of albumin, serum iron, calcium, magnesium, phosphorus, ferritin, B12, folate and $14 \mathrm{FI}$ were also retrieved. All indicators were collected before/during diagnosis, and only the first information was used in case of repeated detection.

\section{Definitions}

The patients were classified into the L1, L2, L3 and L4 types. The L4 lesions were further classified into the EGD, jejunal and proximal ileal subtypes. EGD lesions were defined based on gastroscopy findings and biopsy as follows: (I) erythema, vascular changes, edema, erosions, ulcers, aphthous lesions, and strictures evident in gastroscopy, and (II) focal (discontinuous) and patchy chronic inflammation (lymphocyte and plasma cell infiltration), focal crypt irregularity (discontinuous crypt distortion), granulomas, and irregular villous architecture evident in biopsy $(12,13)$. Jejunal and proximal ileal lesions were defined based on: (I) relevant findings of computed tomography enterography and magnetic resonance enterography, including segmental mural thickening, perienteric infiltration, comb sign, perienteric fistula and/or abscess, segmental bowel stricture, etc. $(14,15)$, and (II) capsule enteroscopy or double balloon enteroscopy findings such as multiple aphthous ulcers measuring $>5 \mathrm{~mm}$ in diameter. Mucosal erythema was not considered as evidence of CD involvement (5).

L4-EGD was defined as the presence of EGD lesions with/without any other gastrointestinal lesions. L4jejunal was defined as jejunal but no EGD involvement, and L4-proximal ileal subtype included patients with proximal ileal involvement without that of jejunal or EGD. Disease behavior was defined based on the findings 
of endoscopy, imageology or surgical specimens. Disease course was defined as time from onset to diagnosis. Perianal complications included perianal fistula, abscess and fissure. The CDAI scores were also evaluated during diagnosis, and CDAI score $\geq 150$ was considered as the active disease state. All complications were recorded during hospitalization before diagnosis. History of surgery was defined as (I) hospital admission due to acute abdominal pain and CD diagnosis during/after operation or (II) CD diagnosis and operation during hospitalization (excluding fistulization).

\section{Enzyme linked immunosorbent assay (ELISA) of FI}

Serum IgG antibodies against tomato, rice, corn, egg, wheat, milk, pork, chicken, beef, crab, codfish, soybean, shrimp and mushroom were detected using specific ELISA kits according to the manufacturer's instructions (Biomerica, Inc., USA). IgG levels $>50 \mathrm{U} / \mathrm{mL}$ was considered positive.

\section{Statistical analysis}

Univariate analyses were performed to compare L4 (and each subtype) and non-L4 patients. All categorical variables were analyzed by the chi-square test, the student $t$-test was used for normally distributed variables and the MannWhitney $U$ test was used for non-normally distributed variables. $P$ value $<0.05$ was considered statistically significant. Random forest technique with python (version 3.8.0) was used for multivariate analysis of the variables that were statistically significant as per univariate analysis. The predictive value of each factor was determined using the "feature_importances" function in the python sklearn package. The metric mean decrease Gini (MDG) was calculated to quantify the contribution of the different factors; higher MDG indicates greater importance. To assess whether a variable increased or decreased the risk for $\mathrm{L} 4$, odds ratios (OR) and 95\% confidence intervals (CI) were estimated using logistic regression.

\section{Results}

\section{Baseline characteristics}

The medical records of 869 patients were collected (Figure S1). The characteristics of the remaining 869 patients are summarized in Table 1. The median disease course was 12 months, and the mean age at onset was 31.6 years. In addition, the median body weight and mean serum albumin levels were $52 \mathrm{~kg}$ and $33.89 \mathrm{~g} / \mathrm{L}$ respectively. Furthermore, 201 patients were smokers, and three-fourths of the cohort $(659 ; 75.83 \%)$ consisted of males. In terms of disease location, only 14 patients had L1 lesions, 130 had L2 lesions, and about a quarter had L3 lesions. More than half the patients $(59.72 \%)$ had L4 lesions, including 335 with proximal ileal, 158 with jejunal and 26 with EGD lesions (Table 2). In addition, $57.54 \%$ of the patients exhibited complications like stricturing and/or invasive disease, and perianal lesions (perianal fistula, abscess, and fissure) were seen in $29.46 \%$ of the patients. Finally, about onetenth $(11.05 \%)$ of the patients developed fever during the hospitalization, and $26.47 \%$ underwent abdominal surgery during hospitalization.

\section{Characteristics of $L 4$ patients}

The proportion of male patients was higher in the L4 group, especially in the proximal ileal and jejunal subtypes (Figure 1A). Likewise, smokers were mainly clustered in the L4 group and the proximal ileal or jejunal subtypes (Figure 1A). However, the age of onset was similar in both L4 and non-L4 groups (Figure 1B). Furthermore, patients with jejunal involvement had a higher incidence of stricturing behavior, and those with jejunal or EGD involvement had a lower incidence of perianal disease (Figure 1C). L4 patients had less complications compared to the non-L4 patients, except for the higher incidence of mouth ulcers in the L4-EGD subtype (Figure 1D). Furthermore, L4 patients were more likely to be overweight, and have higher serum albumin levels at diagnosis (Figure $2 A, B$ ) and a longer disease course, especially with L4-proximal ileal involvement. No significant differences were seen between the two group in terms of abdominal surgery for CD. The data are summarized in Tables S1-S6.

\section{Food intolerance (FI) and other nutritional indicators in CD patients}

A total of 355 patients had complete data of the 14 FI tests conducted between January 2013 and December 2019. The CD patients were most intolerant to corn $(61.10 \%)$, egg $(59.45 \%)$, rice $(59.18 \%)$, tomato $(56.16 \%)$ and soybean $(51.23 \%)$, and least intolerant to cod $(8.77 \%)$, mushroom $(8.49 \%)$, chicken $(4.11 \%)$, pork $(2.47 \%)$ and beef $(1.1 \%)$ (Table 3). Among the L4 patients, corn, rice, tomato, eggs and soybean were the five least tolerated foods. In general, the L4 patients were more likely to show tomato 
Table 1 Basic situation, disease characteristics and complications of L4 patients

\begin{tabular}{|c|c|c|c|c|c|c|c|c|}
\hline \multirow{2}{*}{ Clinical variable } & \multicolumn{2}{|c|}{ All CD patients, $\mathrm{N}=869$} & \multicolumn{2}{|c|}{ L4 patients, $\mathrm{N}=519$} & \multicolumn{2}{|c|}{ Non-L4 patients, $N=350$} & \multirow{2}{*}{$\begin{array}{l}\text { Odds ratio } \\
(95 \% \mathrm{Cl})\end{array}$} & \multirow{2}{*}{$P$ value ${ }^{a}$} \\
\hline & $\mathrm{N}$ & $\%$ & $\mathrm{~N}$ & $\%$ & $\mathrm{~N}$ & $\%$ & & \\
\hline Body weight (kg) & 52.00 & 14.00 & 54.00 & 13.00 & 50.00 & 12.00 & & $0.000^{\star \star}$ \\
\hline Albumin (g/L) & 33.89 & 7.37 & 34.53 & 7.28 & 32.94 & 7.40 & & $0.000^{\star \star}$ \\
\hline Disease course (month) & 12.00 & 33.00 & 12.00 & 43.00 & 9.50 & 22.00 & & $0.001^{\star \star}$ \\
\hline Smoker & 201 & 23.13 & 141 & 27.17 & 60 & 17.14 & $1.80(1.22-2.53)$ & $0.001^{* *}$ \\
\hline \multicolumn{9}{|l|}{ Disease location } \\
\hline L1 (terminal ileum) & 14 & 1.61 & 92 & 17.72 & 14 & 4.00 & $5.17(2.89-9.23)$ & $0.000^{\star *}$ \\
\hline \multicolumn{9}{|l|}{ Disease behavior } \\
\hline B1 (non-stricturing-nonpenetrating) & 369 & 42.46 & 183 & 35.26 & 186 & 53.14 & $0.48(0.36-0.63)$ & $0.000^{\star *}$ \\
\hline B2 (stricturing) & 377 & 43.38 & 257 & 49.52 & 120 & 34.29 & $1.88(1.42-2.48)$ & $0.000^{\star \star}$ \\
\hline B3 (penetrating) & 50 & 5.75 & 29 & 5.59 & 21 & 6.00 & $0.92(0.52-1.65)$ & 0.798 \\
\hline $\mathrm{B} 2$ and B3 & 73 & 8.40 & 50 & 9.63 & 23 & 6.57 & $1.51(0.90-2.53)$ & 0.110 \\
\hline Perianal lesions & 256 & 29.46 & 133 & 25.63 & 123 & 35.14 & $0.63(0.47-0.85)$ & $0.003^{\star \star}$ \\
\hline Disease active period $(C D A I \geq 150)$ & 727 & 83.66 & 424 & 81.70 & 303 & 86.57 & $0.69(0.47-1.01)$ & 0.057 \\
\hline History of surgery & 230 & 26.47 & 139 & 26.78 & 87 & 24.86 & $1.10(0.81-1.50)$ & 0.526 \\
\hline Partial resection of small intestine & 102 & 11.74 & 102 & 19.66 & 0 & & - & - \\
\hline Resection of ileocecum & 26 & 2.99 & 5 & 0.96 & 21 & 6.00 & $0.15(0.05-0.40)$ & $0.000^{\star \star}$ \\
\hline $\begin{array}{l}\text { Partial resection of small intestine }+ \\
\text { resection of ileocecum }\end{array}$ & 4 & 0.46 & 4 & 0.77 & 0 & & - & - \\
\hline Right hemicolectomy & 65 & 7.48 & 18 & 3.46 & 47 & 13.43 & $0.23(0.13-0.40)$ & $0.000^{\star \star}$ \\
\hline $\begin{array}{l}\text { Partial resection of small intestine }+ \\
\text { right hemicolectomy }\end{array}$ & 10 & 1.15 & 10 & 1.93 & 0 & & - & - \\
\hline Left hemicolectomy & 5 & 0.58 & 0 & & 5 & 1.43 & - & - \\
\hline Partial colectomy & 9 & 1.04 & 0 & & 9 & 2.57 & - & - \\
\hline Pancolectomy & 5 & 0.58 & 0 & & 5 & 1.43 & - & - \\
\hline
\end{tabular}

Body weight and disease course were showed as median (IQR). Albumin and age at onset were showed as mean (SD). ${ }^{\text {, }}$, univariate analysis between L4 patients and non-L4 patients, the $\mathrm{P}$ value were showed in table. *, $\mathrm{P}$ value is less than 0.05 , with statistical difference; **, $P$ value is less than 0.01 , with significant statistical difference. CD, Crohn's disease; SD, standard deviation; Gl, gastrointestinal; B2 and B3, patients with both stricturing and penetrating behavior, g/L, gram per liter. 
Table 2 Basic situation, disease characteristics and complications of L4 patients in each subgroup

\begin{tabular}{|c|c|c|c|c|c|c|c|c|}
\hline \multirow{2}{*}{ Clinical variable } & \multicolumn{2}{|c|}{ Non-L4 group, $\mathrm{N}=350$} & \multicolumn{2}{|c|}{ L4-proximal ileal, $\mathrm{N}=335$} & \multicolumn{2}{|c|}{ L4-jejunal, $\mathrm{N}=158$} & \multicolumn{2}{|c|}{ L4-EGD, N=26 } \\
\hline & $\mathrm{N}$ & $\%$ & $\mathrm{~N}$ & $\%$ & $\mathrm{~N}$ & $\%$ & $\mathrm{~N}$ & $\%$ \\
\hline Body weight (kg) & 50.00 & 12.00 & 55.00 & 13.00 & 52.00 & 15.00 & 53.25 & 16.62 \\
\hline Albumin (g/L) & 32.94 & 7.40 & 34.91 & 7.46 & 33.82 & 6.95 & 33.94 & 6.89 \\
\hline Disease course (month) & 9.50 & 22.00 & 12.00 & 43.00 & 12.00 & 30.00 & 10.00 & 69.00 \\
\hline Smoker & 60 & 17.14 & 93 & 27.76 & 41 & 25.95 & 7 & 26.92 \\
\hline \multicolumn{9}{|l|}{ Disease behavior } \\
\hline B1 (non-structuring-nonpenetrating) & 186 & 53.14 & 117 & 34.93 & 52 & 32.91 & 14 & 53.85 \\
\hline $\mathrm{B} 2$ and B3 & 23 & 6.57 & 30 & 8.96 & 19 & 12.03 & 1 & 3.85 \\
\hline Perianal lesions & 123 & 35.14 & 98 & 29.25 & 31 & 19.62 & 4 & 15.38 \\
\hline Disease active period (CDAI $\geq 150$ ) & 303 & 86.57 & 276 & 82.39 & 127 & 80.38 & 21 & 80.77 \\
\hline Other complications & 85 & 24.29 & 45 & 13.43 & 17 & 10.76 & 6 & 23.08 \\
\hline Joint pain & 12 & 3.43 & 0 & 0.00 & 2 & 1.27 & 1 & 3.85 \\
\hline Fever & 55 & 15.71 & 30 & 8.96 & 9 & 5.70 & 2 & 7.69 \\
\hline Mouth ulcer & 14 & 4.00 & 4 & 1.19 & 1 & 0.63 & 3 & 11.54 \\
\hline Right hemicolectomy & 47 & 13.43 & 16 & 4.78 & 1 & 0.63 & 1 & 3.85 \\
\hline $\begin{array}{l}\text { Partial resection of small intestine }+ \text { right } \\
\text { hemicolectomy }\end{array}$ & 0 & & 6 & 1.79 & 4 & 2.53 & 0 & \\
\hline Left hemicolectomy & 5 & 1.43 & 0 & & 0 & & 0 & \\
\hline Partial colectomy & 9 & 2.57 & 0 & & 0 & & 0 & \\
\hline Pancolectomy & 5 & 1.43 & 0 & & 0 & & 0 & \\
\hline
\end{tabular}

Body weight and disease course were showed as median (IQR). Albumin and age at onset were showed as mean (SD). CD, Crohn's disease; SD, standard deviation; EGD, esophago-gastroduodenal; g/L, gram per liter.

intolerance compared to the non-L4 patients (OR 1.65) (Table 3), especially those with proximal ileal or jejunal involvement. In addition, the L4-jejunal patients were more likely to have soybean and rice intolerance compared to the non-L4 patients (Figure 2C). The data are summarized in Tables S7-S10.

L4 patients were more likely to have higher BMI, especially those with L4 proximal ileal involvement, compared to the 

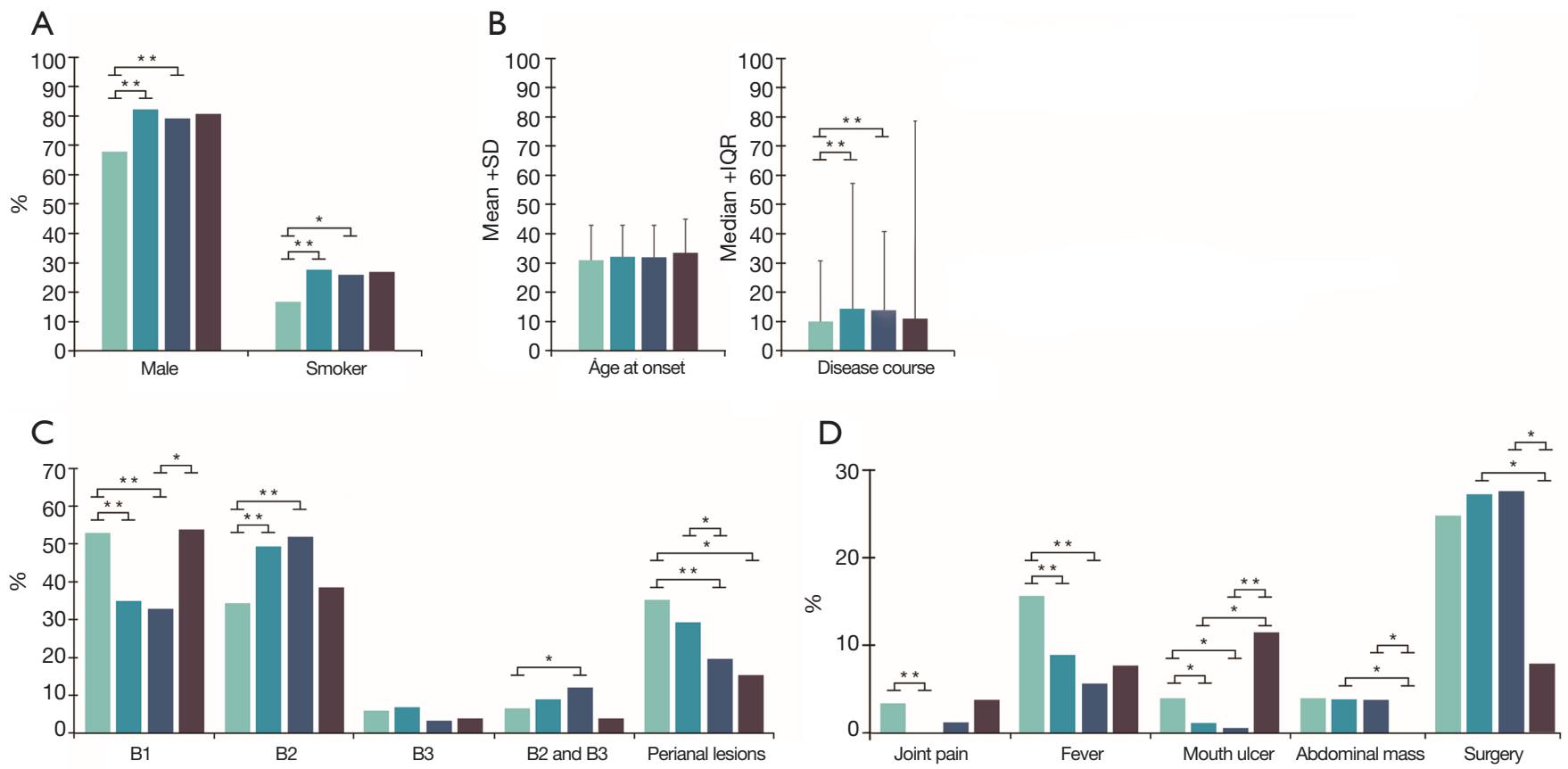

Figure 1 Basic situation, disease characteristics, and complications in L4 patients and each subgroup. (A) Comparison of gender and smoker between L4 patients and each subgroup. (B) Comparison of age at onset and disease course between L4 patients and each subgroup. (C) Comparison of disease behavior and perianal disease between L4 patients and each subgroup. (D) Comparison of disease complications and abdominal surgery of L4 patients and each subgroup. Two sample $t$-test, the Mann-Whitney U test, and chi-square test were used. B1: nonstricturing-nonpenetrating, B2: stricturing, B3: penetrating, B2 and B3: patients with both stricturing and penetrating behavior. *, $\mathrm{P}<0.05$; **, $\mathrm{P}<0.01$. (A,B,C,D) Showed four shades of bars (from left to right: non-L4 patients, L4-proximal ileal, L4-jejunal and L4 EGD). SD, standard deviation; IQR, inter-quartile range; EGD, esophago-gastroduodenal.
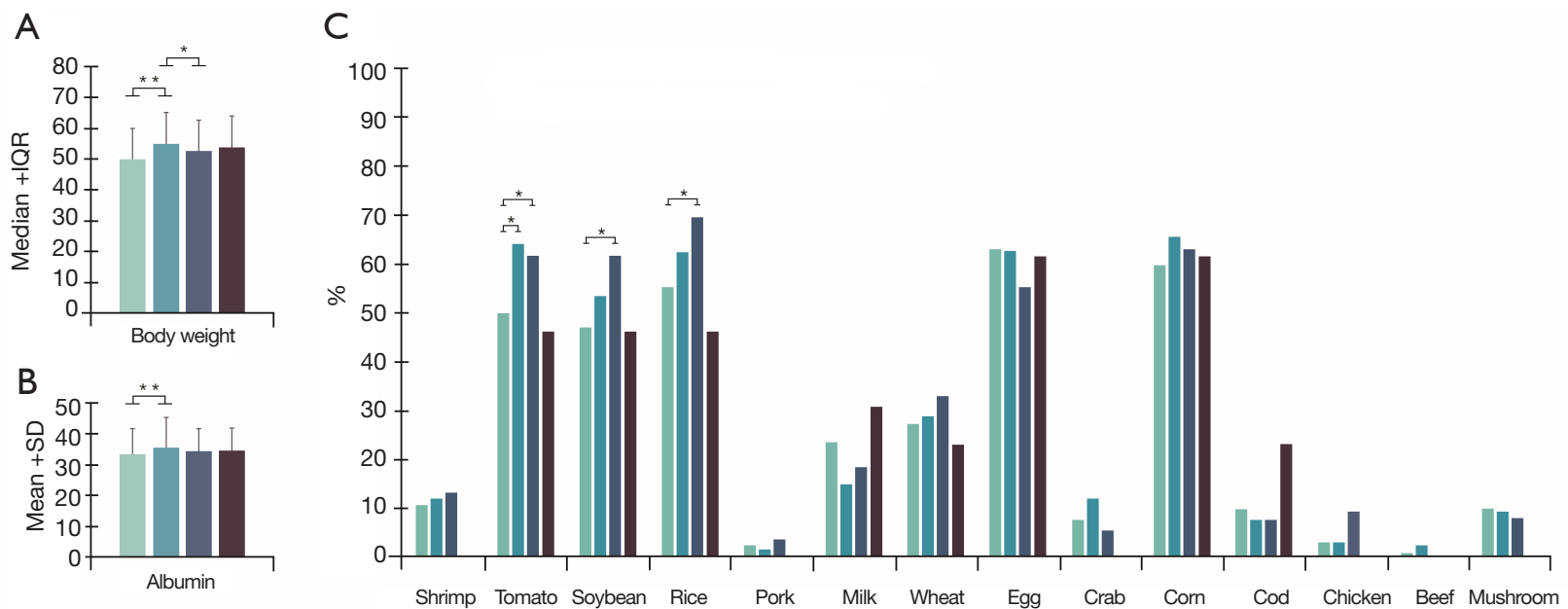

Figure 2 The body weight, albumin, and distribution of 14 kinds of food intolerance in L4 patients and each subgroup. (A) Comparison of body weight between L4 patients and each subgroup. (B) Comparison of albumin between L4 patients and each subgroup. (C) Comparison of 14 kinds of food intolerance between L4 patients and each subgroup. Two sample $t$-test, chi-square test were used. * $\mathrm{P}<0.05$; ${ }^{* *}, \mathrm{P}<0.01$. (A,B,C) Showed four shades of bars (from left to right: non-L4 patients, L4-proximal ileal, L4-jejunal and L4 EGD). SD, standard deviation; IQR, inter-quartile range; EGD, esophago-gastroduodenal. 
Table 3 The distribution of 14 kinds of food intolerance in L4 and non-L4 patients

\begin{tabular}{|c|c|c|c|c|c|c|c|c|}
\hline Food category & \multicolumn{2}{|c|}{ All CD patients, $\mathrm{N}=355$} & \multicolumn{2}{|c|}{ L4 patients, $\mathrm{N}=223$} & \multicolumn{2}{|c|}{ Non-L4 patients, $\mathrm{N}=132$} & Odds ratio $(95 \% \mathrm{Cl})$ & $P$ value \\
\hline Shrimp & 37 & 10.42 & 23 & 10.31 & 14 & 10.61 & $0.96(0.48-1.95)$ & 0.931 \\
\hline Tomato & 205 & 56.16 & 139 & 62.33 & 66 & 50.00 & $1.65(1.07-2.55)$ & $0.023^{*}$ \\
\hline Soybean & 187 & 51.23 & 125 & 56.05 & 62 & 46.97 & $1.44(0.93-2.21)$ & 0.098 \\
\hline Pork & 9 & 2.47 & 5 & 2.24 & 4 & 3.03 & $0.73(0.19-2.78)$ & 0.648 \\
\hline Milk & 69 & 18.90 & 38 & 17.04 & 31 & 23.48 & $0.66(0.39-1.14)$ & 0.138 \\
\hline Wheat & 103 & 28.22 & 67 & 30.04 & 36 & 27.27 & $1.14(0.71-1.84)$ & 0.578 \\
\hline Egg & 217 & 59.45 & 134 & 60.09 & 83 & 62.88 & $0.88(0.57-1.38)$ & 0.602 \\
\hline Cod & 32 & 8.77 & 19 & 8.52 & 13 & 9.85 & $0.85(0.40-1.78)$ & 0.673 \\
\hline Chicken & 15 & 4.11 & 11 & 4.93 & 4 & 3.03 & $1.66(0.51-5.32)$ & 0.389 \\
\hline Beef & 4 & 1.10 & 3 & 1.35 & 1 & 0.76 & $1.78(0.18-17.0)$ & 1.000 \\
\hline Mushroom & 31 & 8.49 & 18 & 8.07 & 13 & 9.85 & $0.80(0.38-1.69)$ & 0.567 \\
\hline
\end{tabular}

*, $P$ value is less than 0.05 , with statistical difference. $C D$, Crohn's disease.

non-L4 patients (Tables S11-S17). In addition, L4 patients were more likely to have higher magnesium and lower ferritin levels (Table S18). No significant differences were seen between both groups in terms of serum iron, calcium, vitamin B12, folate and vitamin D levels (Tables S18-S24).

\section{Relevant factors of L4 CD and subtypes in random forest and logistic regression models}

As shown in Figure $3 A, B, C$, the serum albumin level, body weight and disease course showed higher MDG in random forest for L4 CD and L4-proximal ileal. In logistic regression, body weight (OR 1.054; 95\% CI, 1.024-1.084), disease course (OR 1.010; 95\% CI, 1.001-1.020), stricturing behavior (OR 4.998; 95\% CI, 2.515-9.934) and tomato intolerance (OR 1.313; 95\% CI, 1.043-1.653) were the independent risk factors of the L4 phenotype (Table 4). After adjusting for weight, we found that BMI, disease course, stricturing behavior and tomato intolerance were the independent risk factors of the L4 phenotype (Table S25). The significant factors associated with L4-proximal ileal type included body weight (OR 1.042; 95\% CI, 1.017 1.067; $\mathrm{P}=0.001$ ) and stricturing behavior (OR 3.152; 95\% CI, 1.781-5.580; $\mathrm{P}<0.001$ ), and that for L4-jejunal were stricturing behavior (OR 4.206; 95\% CI, 2.236-8.619; $\mathrm{P}<0.001)$ and perianal disease (OR 0.339; 95\% CI, $0.176-$ $0.653 ; \mathrm{P}<0.001)$.

\section{Discussion}

Improvement in upper gastrointestinal examination techniques has significantly increased the detection rate of the CD L4 phenotype. Indeed, we documented a relatively higher rate of $\mathrm{L} 4$ phenotypes (59.72\%) compared to other studies $(4,6,7,16,17)$. This discrepancy can be attributed to the larger cohort used in our study compared to previous studies on Asian populations $(6,16,17)$, as well as the improvement in imaging techniques for the upper gastric tract over the years. A recent prospective study showed that combining small bowel capsule endoscopy and MRE altered the original Montreal classification in 64\% CD patients (18). We established our diagnosis and classification based on CTE, MRE, double-balloon enteroscopy and capsule endoscopy as appropriate.

The L4 subtypes differ considerably in terms of clinical manifestations and baseline nutritional status. A recent study showed that L4 patients are more likely to present a more complex disease, and those with jejunal involvement 

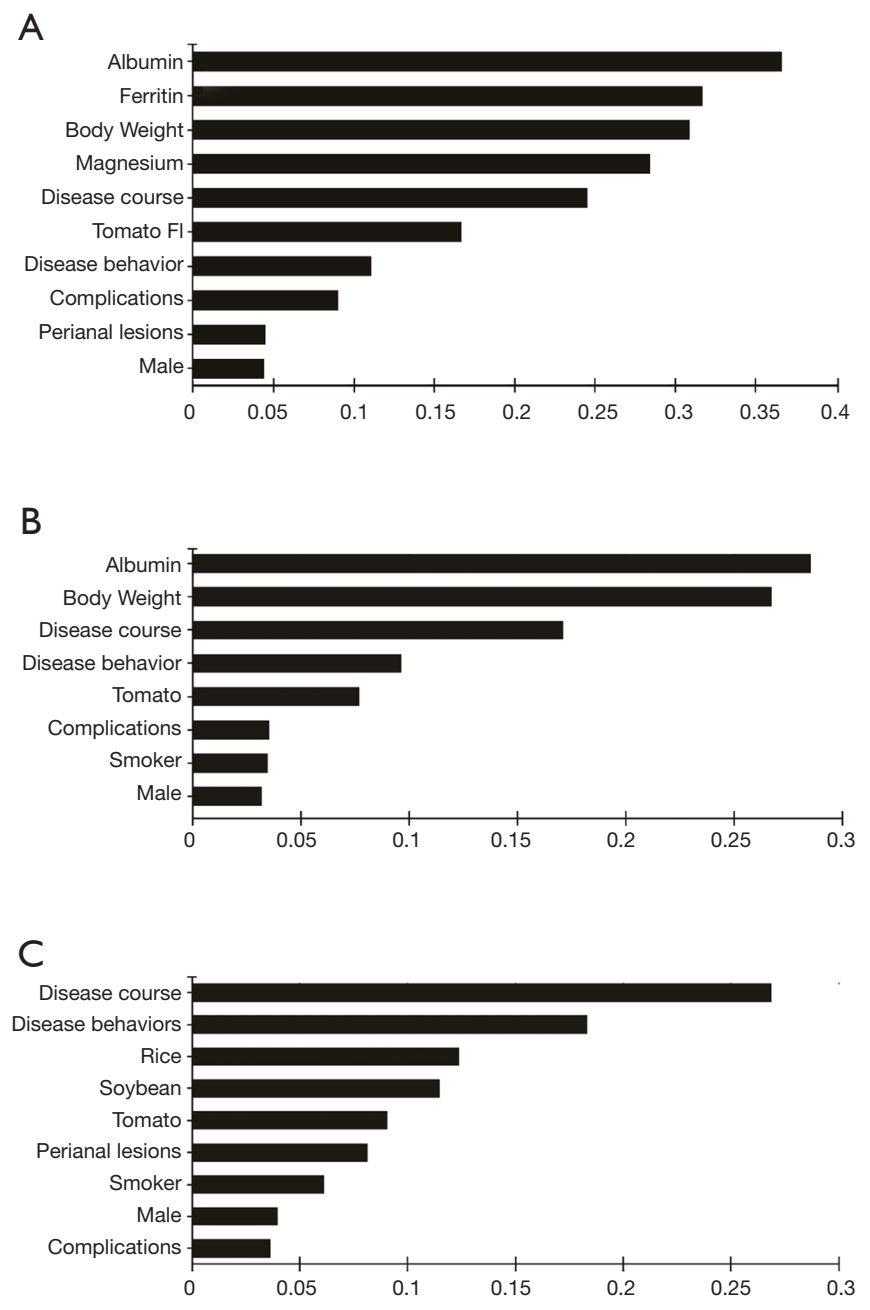

Figure 3 The Random forest important ranking in L4 patients, L4-proximal ileal, and L4-jejunal. (A) The important ranking variables of L4 patients. (B) The important ranking variables of L4-proximal ileal. (C) The important ranking multiple variables of L4-jejunal. We used the "feature_importances" function in the python sklearn package in python to obtain the metric MDG and display from high to low. MDG provides the ways to quantify which factors contribute most to classification accuracy. Greater MDG will indicate that the degree of impurity arising from category could be reduced farthest by one variable, and thus suggests an important associated factor. MDG, mean decrease Gini.

have a higher risk of stricturing (4). In our cohort, both L4-jejunal and proximal ileal patients showed increased stricturing behavior. Nevertheless, the rate of surgical diagnosis was similar between the L4 and non-L4 patients unlike previous reports. Since a higher disease activity correlates to a higher surgical rate (19), we can attribute this observation to the similar disease activity and CDAI scores between both groups. Our study confirmed previous reports showing higher risk of L4 among male patients and smokers, as well as the prolonged disease course of L4 patients (3). In addition, both factors were more prevalent in the proximal ileal and jejunal subtypes.

Several studies have associated L4 with disease relapse, surgical intervention and worse prognosis, but few have reported on the nutritional status of $\mathrm{L} 4$ patients at diagnosis. Body weight and albumin levels are direct indicators of nutritional status. The L4 patients were overweight and had higher albumin levels compared to the 
Table 4 Logistic regression analysis of independent risk factors of L4 patients

\begin{tabular}{llc}
\hline Factors & Odds ratio $(95 \% \mathrm{Cl})$ & $\mathrm{P}$ value \\
\hline Body weight & $1.054(1.024-1.084)$ & 0.000 \\
Disease course & $1.010(1.001-1.020)$ & 0.027 \\
Stricturing behavior & $4.998(2.515-9.934)$ & 0.000 \\
Tomato intolerance & $1.313(1.043-1.653)$ & 0.021 \\
\hline
\end{tabular}

non-L4 patients, especially those with proximal ileal disease. In fact, both factors had the higher MDG value in L4 and L4-proximal ileal patients (Figure $3 A, B$ ). Since weight does not fully represent the nutritional status, we then compared the BMI between L4 patients and non-L4 patients, and found that the former had higher BMI. This is particularly intriguing since inflammation of the small bowel rather than colonic disease leads to protein-energy malnutrition and specific nutrient deficiency (8). There could be several reasons for our contradictory findings. First, food intake is a major factor affecting CD (20), and sweets and fats in particular are associated with CD aggravation (21). These foods are potentially antigenic and can trigger chronic mild inflammation in the gut, leading to changes in nutritional levels (22). Therefore, we also analyzed the FI profile of the L4 patients. Tomato intolerance was an independent risk factor in L4 patients (Tables 3,4), and its potential role in CD needs to be examined further. Second, patients with $\mathrm{CD}$ have an increased risk of developing exocrine pancreatic insufficiency (EPI) (23), which may lead to dyspepsia, malabsorption and malnutrition (23). EPI-induced decrease in the activity of digestive enzymes is related to disease localization and the extent of bowel involvement of CD $(24,25)$. Thus, secondary EPI is a possible reason of the different nutritional levels at diagnosis. Furthermore, heterogenous disease activity may also have resulted aberrantly higher weight and albumin seen in the L4 patients of our cohort. Malnutrition in CD is influenced by several factors, including the activity, duration and extent of the disease, and especially the severity of inflammatory response (11). In fact, recent studies recommend assessment of endoscopy and histological inflammation apart from clinical symptoms in the CD patients $(26,27)$, and chronic inflammation can be a causative factor of the nutritional differences seen in patients at diagnosis.

The large cohort and advanced imaging techniques used in our study resulted in a significantly higher rate of documented L4 disease compared to other studies. We also found that the proximal ileal subtype was predominant in prolonged disease and presence of stricturing behavior. Furthermore, we confirmed a higher nutritional level at diagnosis in the L4 patients, especially those with proximal-ileal involvement. There are however some limitations in our study that ought to be addressed. First, the retrospective design of the study limited the translation of a positive association to causality. Second, body weight, BMI and albumin levels may not be representative of the true nutritional status of CD patients. Finally, despite the apparent role played by FI, the exact pathological relationship between food and CD is not fully clear. Therefore, further prospective trials with multiple nutrition factors and questionnaires should be conducted in order to elucidate the potential associations between CD subtypes and nutritional status.

In conclusion, the L4 phenotype is more common in male patients, and in those with prolonged disease course, stricturing behavior and other complications. In addition, the L4 patients had higher weight and BMI, lower ferritin levels and higher albumin levels at the time of diagnosis, which may be related to FI and disease activity. Our findings provide relevant clinical information for evaluating the nutritional status of CD patients with different phenotypes at the time of diagnosis.

\section{Acknowledgments}

Funding: This work is supported by grants from National Natural Science Foundation of China (81770584).

\section{Footnote}

Reporting Checklist: The authors have completed the STROBE reporting checklist. Available at http://dx.doi. org/10.21037/atm-20-2212

Data Sharing Statement: Available at http://dx.doi. org/10.21037/atm-20-2212

Peer Review File: Available at http://dx.doi.org/10.21037/ atm-20-2212

Conflicts of Interest: All authors have completed the ICMJE uniform disclosure form (available at http://dx.doi. org/10.21037/atm-20-2212). The authors have no conflicts of interest to declare. 


\section{Page 10 of 11}

Ethical Statement: The authors are accountable for all aspects of the work in ensuring that questions related to the accuracy or integrity of any part of the work are appropriately investigated and resolved. The trial was conducted in accordance with the Declaration of Helsinki (as revised in 2013). The research protocol was approved by the Ethical Committee of the Xiangya Hospital of Central South University (No. 201912457) and informed consent was taken from all the patients. The study outcome will not affect the future management of patients. Patient data in this study were retrieved from the hospital medical record system and patient's personal data have been secured.

Open Access Statement: This is an Open Access article distributed in accordance with the Creative Commons Attribution-NonCommercial-NoDerivs 4.0 International License (CC BY-NC-ND 4.0), which permits the noncommercial replication and distribution of the article with the strict proviso that no changes or edits are made and the original work is properly cited (including links to both the formal publication through the relevant DOI and the license). See: https://creativecommons.org/licenses/by-nc-nd/4.0/.

\section{References}

1. Satsangi J, Silverberg MS, Vermeire S, et al. The Montreal classification of inflammatory bowel disease: controversies, consensus, and implications. Gut 2006;55:749-53.

2. Baumgart DC, Sandborn WJ. Crohn's disease. Lancet 2012;380:1590-605.

3. Dulai PS, Singh S, Vande Casteele N, et al. Should We Divide Crohn's Disease Into Ileum-Dominant and Isolated Colonic Diseases? Clin Gastroenterol Hepatol 2019;17:2634-43.

4. Lazarev M, Huang C, Bitton A, et al. Relationship Between Proximal Crohn's Disease Location and Disease Behavior and Surgery: A Cross-Sectional Study of the IBD Genetics Consortium. Am J Gastroenterol 2013;108:106-12.

5. Park SK, Yang SK, Park SH, et al. Long-term prognosis of the jejunal involvement of Crohn's disease. J Clin Gastroenterol 2013;47:400-8.

6. Mao R, Tang RH, Qiu Y, et al. Different clinical outcomes in Crohn's disease patients with esophagogastroduodenal, jejunal, and proximal ileal disease involvement: is L4 truly a single phenotype? Therap Adv Gastroenterol 2018;11:1756284818777938.

7. Sun XW, Wei J, Yang Z, et al. Clinical Features and
Pan et al. Weight, BMI, and FI were linked to L4 CD patients

Prognosis of Crohn's Disease with Upper Gastrointestinal Tract Phenotype in Chinese Patients. Digest Dis Sci 2019;64:3291-9.

8. Goh J, O'Morain CA. Review article: nutrition and adult inflammatory bowel disease. Aliment Pharmacol Ther 2003; 17:307.

9. Grass F, Pache B, Martin D, et al. Preoperative Nutritional Conditioning of Crohn's Patients-Systematic Review of Current Evidence and Practice. Nutrients 2017;9:562.

10. Massironi S, Rossi RE, Cavalcoli FA, et al. Nutritional deficiencies in inflammatory bowel disease: Therapeutic approaches. Clin Nutr 2013;32:904-10.

11. Forbes A, Escher J, Hébuterne X, et al. ESPEN guideline: Clinical nutrition in inflammatory bowel disease. Clin Nutr 2017;36:321-47.

12. Annunziata ML, Caviglia R, Papparella LG, et al. Upper Gastrointestinal Involvement of Crohn's Disease: A Prospective Study on the Role of Upper Endoscopy in the Diagnostic Work-Up. Digest Dis Sci 2012;57:1618-23.

13. Magro F, Langner C, Driessen A, et al. European consensus on the histopathology of inflammatory bowel disease. J Crohns Colitis 2013;7:827-51.

14. Yamagami H, Watanabe K, Kamata N, et al. Small Bowel Endoscopy in Inflammatory Bowel Disease. Clin Endosc 2013;46:321.

15. Takenaka K, Ohtsuka K, Kitazume Y, et al. Comparison of Magnetic Resonance and Balloon Enteroscopic Examination of the Small Intestine in Patients With Crohn's Disease. Gastroenterology 2014;147:334-342.e3.

16. Leong RW, Lau JY, Sung JJ. The epidemiology and phenotype of Crohn's disease in the Chinese population. Inflamm Bowel Dis 2004;10:646-51.

17. Song XM, Gao X, Li MZ, et al. Clinical features and risk factors for primary surgery in 205 patients with Crohn's disease: analysis of a South China cohort. DIS Colon Rectum 2011;54:1147-54.

18. Greener T, Klang E, Yablecovitch D, et al. The Impact of Magnetic Resonance Enterography and Capsule Endoscopy on the Re-classification of Disease in Patients with Known Crohn's Disease: A Prospective Israeli IBD Research Nucleus (IIRN) Study. J Crohns Colitis 2016;10:525-31.

19. Rinawi F, Assa A, Hartman C, et al. Incidence of Bowel Surgery and Associated Risk Factors in Pediatric-Onset Crohn's Disease. Inflamm Bowel Dis 2016;22:2917-23.

20. Hou JK, Lee D, Lewis J. Diet and Inflammatory Bowel Disease: Review of Patient-Targeted Recommendations. Clin Gastroenterol Hepatol 2014;12:1592-600. 
21. Sakamoto N, Kono S, Wakai K, et al. Dietary risk factors for inflammatory bowel disease: a multicenter case-control study in Japan. Inflamm Bowel Dis 2005;11:154-63.

22. Banai J. Nutrition in inflammatory bowel disease. Orv Hetil 2009; 150:839-45.

23. Singh VK, Haupt ME, Geller DE, et al. Less common etiologies of exocrine pancreatic insufficiency. World J Gastroenterol 2017;23:7059-76.

24. Hegnhøj J, Hansen CP, Rannem T, et al. Pancreatic function in Crohn's disease. Gut 1990;31:1076-9.

25. Winter TA, O'Keefe SJ, Callanan M, et al. Impaired

Cite this article as: Pan J, Fu D, Li Y, Wang Y, Lian G, Liu X. Body weight, serum albumin and food intolerance were linked to upper gastrointestinal Crohn's disease: a 7-year retrospective analysis. Ann Transl Med 2020;8(21):1370. doi: 10.21037/atm-202212 gastric acid and pancreatic enzyme secretion in patients with Crohn's disease may be a consequenece of a poor nutritional state. Inflamm Bowel Dis 2004;10:618-25.

26. Danese S, Sandborn WJ, Colombel J, et al. Endoscopic, Radiologic, and Histologic Healing With Vedolizumab in Patients With Active Crohn's Disease. Gastroenterology 2019;157:1007-1018.e7.

27. Carman N, Tomalty D, Church PC, et al. Clinical disease activity and endoscopic severity correlate poorly in children newly diagnosed with Crohn's disease. Gastrointest Endosc 2019;89:364-72. 